\title{
$\begin{array}{ll}\text { Research Square } & \begin{array}{l}\text { Preprints are preliminary reports that have not undergone peer review. } \\ \text { They should not be considered conclusive, used to inform clinical practice, } \\ \text { or referenced by the media as validated information. }\end{array}\end{array}$
}

\section{Comprehensive Analysis of miRNA-mRNA/IncRNA During Gonadal Development of Triploid Rainbow Trout (Oncorhynchus mykiss)}

\author{
Tianqing Huang \\ Chinese Academy of Fishery Sciences

\section{Wei Gu} \\ Chinese Academy of Fishery Sciences \\ Enhui Liu \\ Chinese Academy of Fishery Sciences \\ Xiulan Shi \\ Chinese Academy of Fishery Sciences \\ Bingqian Wang \\ Chinese Academy of Fishery Sciences \\ Gefeng Xu ( $\nabla$ xugefeng@hrfri.ac.cn) \\ Chinese Academy of Fishery Sciences \\ Zuochun Yao \\ Chinese Academy of Fishery Sciences
}

Research article

Keywords: Triploid, fertility, gonadal development, rainbow trout, IncRNA, miRNA, ceRNA

Posted Date: August 4th, 2020

DOI: https://doi.org/10.21203/rs.3.rs-31357/v1

License: (c) (i) This work is licensed under a Creative Commons Attribution 4.0 International License. Read Full License

Version of Record: A version of this preprint was published at Genomics on November 1st, 2021. See the published version at https://doi.org/10.1016/j.ygeno.2021.08.018. 


\section{Abstract}

Background: Polyploidy is an important force to improve biological evolution. Chromosomal ploidy manipulation is one of the means to create excellent germplasm. It has an important significance to understand the effect of genome duplication on fertility in polyploidy breeding. In this study, coding and non-coding RNAs involved in gonadal development were characterized, and their relationships were explored.

Results: By high-throughput sequencing, we compared the expression profiles of gonadal mRNA, long non-coding RNA (IncRNA), and microRNA (miRNA) at different developmental stages [65 days post fertilisation (dpf), $180 \mathrm{dpf}$, and $600 \mathrm{dpf}$ ] between the diploid (XX) and triploid (XXX) rainbow trout. A majority of differentially expressed (DE) RNAs was screened. The overlapped DE mRNAs of three stages were functionally annotated by Gene Ontology (GO) and Kyoto Encyclopaedia of Genes and Genomes (KEGG) pathway analysis. According to the overlapped miRNAs, the predicted miRNA-mRNA/IncRNA network was constructed based on the target pairs of IncRNA-miRNA and mRNA-miRNA. Also, RT-qPCR was performed to validate the credibility of the network.

Conclusions: Lots of IncRNAs involved in fertility were characterised by this network, which provided a reference sequence for further research. In summary, this study explored the potential interplay between coding and noncoding RNAs during the gonadal development of polyploid fish and elaborated the effect of triploidization on fertility.

\section{Background}

The genome polyploidization provide favorable opportunity for new genes and traits formation of species, which is one of the main driving forces for species evolution (Comai, 2005; Leitch and Leitch, 2008; Van de Peer et al., 2009; 2017). The teleost-specific genome duplication is considered to be the major driver for species sharp increase and rapid radiation of teleost (Jaillon et al., 2004; Meyer and Van de Peer, 2005; Braasch et al., 2016). More strikingly, most triploid animal has been reported to be sterile because of the imbalance of nuclear-cytoplasmic ratio, the disorders of homologous chromosome pairing during meiosis or the epigenetic instability (Comai, 2005), which would lead to produce abnormal gametes and the cessation of gonad development (Benfey, 1999; Tiwary et al., 2004). Infertility is often manifested in artificial aquatic animals, such as triploid oyster and rainbow trout (Guo et al., 1996; Huang et al., 2017), and it provides the greater advantages in aquaculture. Especially for the salmonidae, due to the characteristics of mass mortality in breeding season, the infertility could avoid death and energy loss, which would provide large-size products. However, the induced tetraploid rainbow trout could produce functional gametes resulted from pairing of homoeologous chromosomes (Chourrout et al., 1986; Diter et al., 1988). So the regulatory mechanism of triploidization on sterility at the transcriptional or post-transcriptional level should be studied further.

IncRNAs, with lengths longer than 200 nucleotides, are devoid of open reading frames (ORFs) and are often polyadenylated (Lau, 2014). The importance of IncRNAs has been immensely underestimated in earlier studies because of their low expression and low sequence conservation as compared to the mRNAs, and their designation as a transcriptional noise (Wu et al., 2014). Accumulating evidence indicates that IncRNAs play critical roles in various biological processes in animals and plants. The biological functions of IncRNAs are extremely complex. Multiple studies have shown that IncRNA participate in many biological processes, such as sexual reproduction (Zhang et al., 2014), gonadal development (Taylor et al., 2015), and gametogenesis (Herrera et al., 2005; Laiho et al., 2013). Bioinformatic analysis of Amur sturgeon (Zhang et al., 2019) and Chinese softshell turtle (Zhang et al., 2018) gonads have shown that the expression of the majority of IncRNAs is significantly different between males and females. Target genes prediction has shown IncRNAs could regulate many genes responsible for sex determination and gametogenesis (Wen et al., 2016). Research in Drosophila has demonstrated that IncRNAs play an integral role in spermatogenesis and development. Through high-throughput sequencing and analysis of Drosophila in different tissues at different developmental stages, Brown has found that 125 IncRNAs are specifically expressed in the testis (Brown et al., 2014). Wen knocked out 105 IncRNAs which are specifically expressed in Drosophila testis and found that partial or complete loss of fertility could occur if 33 IncRNAs are knocked out (Wen et al., 2016).

miRNA could specifically target mRNA of the genes to regulate gene expression, and exerts important regulatory functions in various biological processes, such as growth, differentiation, apoptosis, signal transduction, and production. In fish, miRNA could regulate gonadal development and germ cell meiosis. The expression profiles in many fishes have shown significant differences in the ovary and testis (Xiao et al., 2014). miR-200b expression in flounder ovary was found to be seven-fold higher than that in testis, while Pol-miR-726 expresses specifically in the ovary of flounder (Gu et al., 2014). miR-430, miR-21, let-7, miR-181a, and miR-143 play important roles in the differentiation and development of ovary in Cyrinus carpio. miR-143 exerts the same function as that in mammals (Wang et al., 2017) signifying that the miRNAs are highly conserved during species evolution. In zebrafish, the expression of miR-17a and miR-430b in the follicles gradually reduces with the development of follicles (Abramov et al., 2013). Hence, miRNAs could negatively regulate the maturity of fish follicles. Germinal vesicle breakdown and meiosis of oocytes are regulated by the synthesis and degradation of maturation-promoting factor (MPF), cyclin B, and cdc2 kinase complex (Pati \& Habibi, 2000). miR-2 and miR-133 can efficiently inhibit the expression of cyclin B, which is important for oocytes entering the process of the second meiosis (Song et al., 2014). It is essential to ascertain the functions of miRNAs and the interactions with their targeted genes for understanding the mechanism of gonadal differentiation and development in fish.

By high-throughput sequence and analysis, this study used differentially expressed (DE) mRNAs, miRNAs, and IncRNAs between diploid (XX) and triploid (XXX) rainbow trout gonads at different developmental stages. Furthermore, and the functions of DE RNAs were analysed. The predicted 
miRNA-mRNA/IncRNA network regulating gonadal development was constructed based on miRNA-mRNA and miRNA-IncRNA pairs. This research would provide a better understanding of the effects of genome duplication on fertility, and also lay the necessary foundation for the industry involved in ploidy breeding and fertility regulation.

\section{Results}

\section{Identification of DE mRNAs, DE IncRNAs, and DE miRNAs during gonadal development}

According to a previous study, the key point of gonadal differentiation of rainbow trout is 35-70 dpf (Feist \& Schreck, 1996). The gonadal development of triploid rainbow begins to show abnormal characteristics after $180 \mathrm{dpf}$ (Xu et al., 2016). So, gonadal tissues of $65 \mathrm{dpf}$, $180 \mathrm{dpf}$, and $600 \mathrm{dpf}$ were chosen for transcriptome sequencing. The principal component analysis (PCA) plot of our samples was as shown in Supplementary Fig. S2. Transcripts from gonads of the same developmental period but different biological replicates tended to cluster together. As compared to the diploid (XX), the results showed that 425 DE mRNAs at $65 \mathrm{dpf}$ (244 up and 181 down), 1, 022 DE mRNAs at $180 \mathrm{dpf}$ (640 up and 382 down), and 2 , $408 \mathrm{DE}$ mRNAs at $600 \mathrm{dpf}$ (1862 up and 546 down) were identified in the triploid (XXX) (Fig. 1A-C). The overlapped DE mRNAs between two developmental periods were 124, and among three periods were 3 (Fig. 1J). For IncRNAs, 102 DE IncRNAs at 65 dpf (53 up and 49 down), 226 DE IncRNAs at $180 \mathrm{dpf}$ (129 up and 97 down), and 1, 329 DE IncRNAs at $600 \mathrm{dpf}$ (1, 081 up and 248 down) were identified (Fig. 1D-F). Two DE IncRNAs were overlapped among the three periods and $38 \mathrm{DE}$ IncRNAs were overlapped between two developmental periods (Fig. 1K). The detail information of the overlapped IncRNAs is shown in Table 1. For miRNAs, 39 DE miRNAs at $65 \mathrm{dpf}$ (23 up and 16 down), 79 DE miRNAs at $180 \mathrm{dpf}$ ( $21 \mathrm{up}$ and 58 down), and 42 DE miRNAs at $600 \mathrm{dpf}$ (28 up and 14 down) were characterised (Fig. 1G-I). The overlapped DE miRNAs between 65 dpf and $180 \mathrm{dpf}$ were 4, and 5 DE miRNAs were overlapped between $180 \mathrm{dpf}$ and $600 \mathrm{dpf}$ (Fig. 1L). The sequences of the overlapped DE miRNAs are shown in Table 2. The full DE reports of ballgown runs for each class of transcripts were provided in the supplementary information (Supplementary Table S5-S13). Both mRNA and IncRNA DEGs were retrieved based on q-value $<0.05$, whereas miRNA ones on p-value $<0.05$.

\section{Functional analysis of DE mRNAs, DE IncRNAs, and DE miRNAs during gonadal development}

GO analysis indicated that the differentially expressed mRNAs at each time point were distributed in biological process (BP), cellular component (CC) and molecular function (MF) classifications (Fig. 2). As for up-regulated DE mRNAs of $65 \mathrm{dpf}$, the most significant enriched BP terms were transport, regulation of transcription and oxygen transport. Pathway analysis demonstrated that the up-regulated mRNAs of 65 dpf were enriched in PPAR signaling pathway, adipocytokine signaling pathway and Notch signaling pathway (Fig. 3A). The most enriched BP terms of down-regulated DE mRNAs of $65 \mathrm{dpf}$ were lipid transport, one-carbon metabolic process and response to type 1 interferon. KEGG pathway analysis revealed that downregulated DE mRNAs of $65 \mathrm{dpf}$ were most enriched in NOD-like receptor signaling pathway, influenza A and nitrogen metabolism (Fig. 3B). GO analysis showed that the most enriched BP terms of up-regulated DE mRNAs of $180 \mathrm{dpf}$ were in regulation of transcription, signal transduction and multicellular organism development. As for the down-regulated mRNAs of $180 \mathrm{dpf}$, the most BP terms were enriched in cell adhesion, cell-matrix adhesion, homophilic cell adhesion via plasma membrane adhesion molecules, response to bacterium. Pathway analysis showed that the upregulated mRNAs of $180 \mathrm{dpf}$ were most enriched in focal adhesion, vascular smooth muscle contraction and ECM-receptor interaction (Fig. 3C), while the down-regulated mRNAs of $180 \mathrm{dpf}$ were most in Calcium signaling pathway, Salivary secretion, Fat digestion and absorption, Phenylalanine, tyrosine and tryptophan biosynthesis (Fig. 3D). As for DE mRNAs at $600 \mathrm{dpf}$, GO analysis demonstrated that up-regulated and downregulated mRNAs were all most enriched in regulation of transcription-DNA templated, transport and signal transduction of BP terms. KEGG pathway analysis revealed that up-regulated mRNAs were enriched in neuroactive ligand-receptor interaction, cell adhesion molecules (CAMs), calcium signaling pathway, ECM-receptor interaction and Hipoo signalling pathway (Fig. 3E), and down-regulated mRNAs were enriched in lysosome, complement and coagulation cascades, PPAR signaling pathway and Ras signaling pathway (Fig. 3F). GO enrichment analysis showed that the overlapped DE mRNAs were mostly enriched in regulation of transcription, DNA template, protein phosphorylation, regulation of Rho protein signal transduction, cell adhesion and regulation of GTPase activity of BP terms. The most significantly enriched CC terms were cytoplasm, integral component of membrane and membrane. The most significantly enriched MF terms were metal ion binding, zinc ion binding and ATP binding (Fig. 4). The KEGG analysis showed that the most enriched pathways were RNA degradation and vascular smooth muscle contraction (Table 3 ). The 38 overlapped DE IncRNAs had unknown functions. As for the overlapped DE miRNAs, miR-26a (Kang et al., 2017) and miR-1388 (Yang et al., 2018) have been proven to be correlated with gonadal development in fish.

\section{Construction of ceRNA network of miRNA-mRNA/IncRNA}

Three predicted miRNA-mRNA/IncRNA interaction networks with up- and down-regulated nodes had been established for each developmental stage, which was integrated from the relationship between DE IncRNA-miRNA and DE mRNA-miRNA using perl scripts. The information including all the above interactions was imported into Cytoscape to construct predicted miRNA-mRNA/IncRNA interaction networks. The network of 65 dpf consisted of 10 miRNAs, 13 IncRNAs and 13 mRNAs (Fig. 5A). The network of 180 dpf was composed of 45 miRNAs, 50 IncRNAs and 100 mRNAs (Fig. 5B).

Page $3 / 20$ 
The predicted network of $600 \mathrm{dpf}$ was more complex, which consisted of 33 miRNAs, 102 IncRNAs and 135 mRNAs (Fig. 5C). According the functional enrichment analysis of DE mRNAs in each developmental stage, the establishment of miRNA-mRNA/IncRNA interaction networks was helpful to predict the regulatory roles of some non-coding RNAs in fish fertility. The overlapped DE miRNAs of three developmental stages were used to construct a network of miRNA-mRNA/IncRNA, which consisted of 43 transcripts (6 miRNAs, 12 IncRNAs, and 25 mRNAs) (Fig. 6).

\section{Validation of DE miRNAs, DE IncRNAs, and DE mRNAs by qPCR}

To verify the credibility of sequencing, 7 miRNAs, 10 IncRNA, and 10 mRNAs were chosen randomly from the RNA-seq. The expression of different ploidy at different developmental stages of these RNAs was detected by RT-PCR. The results show that the relative expression of 7 miRNAs, 10 IncRNA, and 10 mRNAs in triploid (XXX) was consistent with the sequencing results (Supplementary Table S14), which was compared to the diploid (XX) (Fig. 7). It was also demonstrated that the IncRNA-miRNA-mRNA network was credible, and could guide on the relationship between ploidy manipulation and fertility. 
Table 1

Overlapping DE IncRNAs during gonadal development between the diploid $(X X)$ and triploid $(X X X)$ rainbow trout.

\begin{tabular}{|c|c|c|c|c|}
\hline LncRNA & Strand & chromosome & Exons & Length (bp) \\
\hline MSTRG.78113.2 & - & chrNW_018566344.1 & 2 & 1446 \\
\hline MSTRG.17574.1 & - & $\operatorname{chr} 15$ & 1 & 791 \\
\hline MSTRG.21358.7 & + & chr2 & 2 & 4007 \\
\hline MSTRG.29187.3 & - & $\operatorname{chr19}$ & 2 & 1496 \\
\hline MSTRG.8660.2 & - & $\operatorname{chr} 12$ & 2 & 1676 \\
\hline MSTRG.32111.1 & - & chr2 & 2 & 2814 \\
\hline MSTRG.82591.5 & + & chrNW_018591615.1 & 3 & 909 \\
\hline MSTRG.59623.1 & - & chr6 & 2 & 834 \\
\hline MSTRG.12993.1 & - & chr13 & 1 & 578 \\
\hline MSTRG.65152.1 & - & chr8 & 1 & 550 \\
\hline MSTRG.14693.2 & + & chr14 & 3 & 912 \\
\hline MSTRG.20487.2 & - & chr16 & 2 & 3067 \\
\hline MSTRG.25106.2 & - & $\operatorname{chr18}$ & 2 & 1704 \\
\hline MSTRG.28943.3 & - & $\operatorname{chr19}$ & 2 & 1467 \\
\hline MSTRG.60464.1 & + & chr7 & 2 & 1525 \\
\hline MSTRG.13824.2 & + & $\operatorname{chr13}$ & 2 & 2423 \\
\hline MSTRG.35355.2 & + & chr21 & 2 & 3887 \\
\hline MSTRG.40806.1 & - & chr25 & 3 & 818 \\
\hline MSTRG.27463.5 & + & chr19 & 2 & 926 \\
\hline MSTRG.73462.2 & - & chrNW_018544540.1 & 2 & 246 \\
\hline MSTRG.40175.2 & + & chr25 & 2 & 1953 \\
\hline MSTRG.73961.4 & - & chrNW_018546694.1 & 3 & 1130 \\
\hline MSTRG.73961.2 & - & chrNW_018546694.1 & 2 & 1163 \\
\hline MSTRG.52105.1 & - & chr4 & 2 & 2801 \\
\hline MSTRG.33565.5 & + & chr20 & 4 & 1255 \\
\hline MSTRG.9390.1 & + & $\operatorname{chr} 12$ & 1 & 5466 \\
\hline MSTRG.73462.1 & - & chrNW_018544540.1 & 2 & 289 \\
\hline MSTRG.33565.1 & + & chr20 & 4 & 1028 \\
\hline MSTRG.52608.3 & - & chr4 & 2 & 2771 \\
\hline MSTRG.77434.2 & + & chrNW_018563583.1 & 2 & 418 \\
\hline MSTRG.63835.1 & - & chr8 & 3 & 1337 \\
\hline MSTRG.73962.1 & + & chrNW_018546694.1 & 1 & 1232 \\
\hline MSTRG.19305.1 & + & $\operatorname{chr} 16$ & 2 & 1408 \\
\hline MSTRG.74275.3 & - & chrNW_018548194.1 & 2 & 1797 \\
\hline MSTRG.33257.1 & - & chr20 & 1 & 377 \\
\hline MSTRG.4797.1 & - & $\operatorname{chr} 10$ & 1 & 434 \\
\hline MSTRG.80034.1 & + & chrNW_018575822.1 & 2 & 382 \\
\hline MSTRG.65644.1 & - & chr8 & 1 & 209 \\
\hline
\end{tabular}


Table 2

Overlapped DE miRNAs during gonadal development between the diploid (XX) and triploid $(X X X)$ rainbow trout

\begin{tabular}{|lll|}
\hline MicroRNA & $\boldsymbol{p}$ value & Sequence \\
\hline ssa-miR-730a-5p & 0.0495 & TCCTCATTGTGCATGCTGTGTG \\
\hline dre-miR-26a-5p_R+2_1ss17TG & 0.0043 & TTCAAGTAATCCAGGAGAGGCTTT \\
\hline ola-miR-199a-3p_L+1R+1 & 0.0198 & AACAGTAGTCTGCACATTGGTTAT \\
\hline gmo-miR-10544-3p_R+1_1ss18CT & 0.0031 & TGCGCACGGGGCCACGCTCTGC \\
\hline PC-5p-21854_317 & 0.0137 & TGGATACAATTGAGTTTTGGAT \\
\hline oni-miR-1388_1ss1AT & 0.0024 & TGGACTGTCCAACCTGAGAATG \\
\hline PC-5p-63051_86 & 0.0346 & TTCTTCGTCTGTAGGATTCAAATGTA \\
\hline PC-5p-28329_238 & 0.0466 & CCCTGTTGTACTTCTCCTGC \\
\hline PC-5p-10869_636 & 0.0263 & TGAGAACTAGCGCACCATTGGT \\
\hline
\end{tabular}

Table 3

KEGG pathway enrichment of the overlapped DE mRNAs

\begin{tabular}{|c|c|c|c|}
\hline Pathway ID & Pathway Name & Genes & $p$ value \\
\hline \multirow[t]{6}{*}{3018} & \multirow[t]{6}{*}{ RNA degradation } & XM_021622228.1;XM_021620588.1;XM_021620590.1; & \multirow[t]{6}{*}{0.03} \\
\hline & & XM_021620585.1;XM_021620589.1;XM_021620586.1; & \\
\hline & & XM_021620591.1;XM_021620587.1;XM_021589586.1; & \\
\hline & & XM_021589590.1;XM_021589585.1;XM_021589591.1; & \\
\hline & & XM_021589592.1;XM_021589588.1;XM_021589583.1; & \\
\hline & & XM_021589584.1 & \\
\hline \multirow[t]{6}{*}{4270} & \multirow[t]{6}{*}{ Vascular smooth muscle contraction } & XM_021607281.1;XM_021607280.1;XM_021607278.1; & \multirow[t]{6}{*}{0.03} \\
\hline & & XM_021607274.1;XM_021607276.1;XM_021607275.1; & \\
\hline & & XM_021607277.1;XM_021569166.1;XM_021569164.1; & \\
\hline & & XM_021569167.1;XM_021569165.1;XM_021621261.1; & \\
\hline & & XM_021621262.1;XM_021621263.1;XM_021617669.1; & \\
\hline & & XM_021617668.1;XM_021617666.1;XM_021617667.1 & \\
\hline
\end{tabular}

\section{Discussion}

Polyploidy plays an important role in biological evolution. Compared to diploids, polyploids must overcome genetic instability and meiosis abnormalities after genome duplication (Stenberg \& Saura, 2013). Gonadal development in polyploids is an extremely complex process and involves formation, migration, and differentiation of the reproductive cells (Cifuentes et al., 2010). In this study, we selected gonadal tissues at the key time points in diploid (XX) and triploid (XXX) Oncorhynchus mykiss, which were of $65 \mathrm{dpf} 180 \mathrm{dpf}$, and $600 \mathrm{dpf}$. The expression of the RNAs at these three-time points might be used to examine the effects of genome duplication on gonadal development. There are still many unknown functions of IncRNAs and miRNAs in regulating gonadal development. According to ceRNA regulatory mechanisms, gene-encoding mRNA transcripts contain many microRNA response elements (MREs). miRNAs can bind to the mRNAs through MREs to cause mRNA degradation or inhibit its translation (Ye et al., 2008). When IncRNA and mRNA contain the same MREs, they compete for the same type of miRNAs. Hence, IncRNAs indirectly regulate the expression of mRNAs through MREs, thereby regulating their biological functions (Guo et al., 2014). This study constructed effector networks of the three RNA species based on the above reasons for a more in-depth understanding of the effects of ploidy on gonadal development.

Among these enriched pathways, the Hippo pathway plays an important role in regulating follicle development (Kawashima \& Kawamura, 2018). The Hippo signalling pathway contains many negative regulatory factors and can inactivate the transcription cofactor YAP and the binding motif TAZ (Halder \& Johnson, 2011). Filamentous-actin (F-actin) can disrupt the Hippo signalling pathway, thereby inducing synthesis of connective tissue growth factor and the anti-apoptotic BIRC (Pan, 2007). This causes YAP to be localised inside the nucleus and further stimulates the growth of secondary follicles in vivo and in vitro (Kawamura et al., 2013). In addition, persistent YAP overexpression in human and mouse ovaries can promote follicle growth (Cheng et al., 2015). In this study, sox19b and sox21b in the Hippo signalling pathway were enriched. Sox 19 was overexpressed in the 
gonads of Dicentrarchus labrax, and its expression in the ovaries was higher than that in the testes. Sox19 plays an important role in ovarian differentiation (Navarro-Martin et al., 2012). Based on these findings, Hippo signaling pathways may be associated with the development of the haploid gonads of rainbow trout.

Peroxisome proliferator-activated receptors (PPARs) are important in the reproductive system and are expressed at different levels in the hypothalamic-pituitary-gonadal (HPG) axis (Bogacka et al., 2015). Some studies have demonstrated that PPARs can regulate ovum proliferation, tissue remodelling, and hormone synthesis (Barak et al., 2002). In this study, the biological analysis showed that the differentially expressed genes (DEGs) which were enriched in the PPAR signalling pathway included matrix metalloproteinase 13 (MMP13), which upregulated at 65 dpf and 180 dpf, but downregulated at $600 \mathrm{dpf}$. MMP13 is mainly expressed in the primary follicles and plays an important role in follicle development along with MMP1 (Fujihara et al., 2016). The study found that PLIN1 was specifically expressed in the testis and its mRNA level decreased significantly with development, which was confirmed to play an important role in the early stage of the first meiosis of spermatogenesis (Chen et al., 2014). Interestingly, in this study, PLIN1 upregulated at 180dpf, but downregulated at $600 \mathrm{dpf}$. Moreover, fa2h expression was upregulated at $180 \mathrm{dpf}$, fa2h mouse homologs were confirmed to be involved in sperm formation (Florencia X, et.al, 2018). Taken together, this result suggests that PPARs pathways may affecting polyploid infertility of rainbow trout. Genes enriched in the Wnt signalling pathway include wnt9a (180dpf upregulated) and ctnnb1 (600dpf upregulated). The WNT/CTNNB1 signalling pathway involves many important biological processes, including sex determination, and reproductive system specialisation (Chassot et al., 2008; Mark et al., 2008). In male gonads in mice, the RSP01 protein can activate the WNT/CTNNB1 signalling pathway to enhance PGC specialisation (Chassot et al., 2011). Overexpression of the WNT/CTNNB1 signalling pathway could downregulate the specialisation of spermatogonia and cause spermatocyte apoptosis, ultimately resulting in testicular hypoplasia (Chassot et al., 2017). The predicted miRNA-mRNA/IncRNA interaction network based on overlapped DE miRNAs included important genes in the WNT pathway, showing that the WNT pathway plays an important role in regulating polyploid infertility, and further provides a direction for future studies.

The Venn diagram shows that the overlapped DE protein-encoding genes with significant differences during the three developmental stages of 65 dpf, $180 \mathrm{dpf}$, and $600 \mathrm{dpf}$. The functions of these genes were characterised and the significantly enriched GO and KEGG pathways were identified which were intimately associated with regulation of transcription and signal transduction. The overlapped DE miRNAs in the predicted miRNAmRNA/IncRNA interaction network constructed in this study showed significant differential expression in the ovaries from diploids (XX) and triploids $(X X X)$ at different development stages, implying that these miRNAs play a role in the normal development of gonads. Among the identified miRNAs, many are worthy of further studies. According to previous studies, miR-26a can regulate gonadal development in Paralichthys olivaceus through its target, the em $x 2$ gene (Yin et al., 2015). miR-26a-5p overexpression can inhibit TNRC6A expression and promote the proliferation of the theca cells (Kang et al., 2017). miR-1388 plays an indispensable role in regulating gonadal development in $P$. olivaceus through its target, the nectin21 gene (Yang et al., 2018). The role of these IncRNAs, mRNAs, and miRNAs from this predicted ceRNA network in gonadal development and meiosis of reproductive cells requires further in-depth research.

\section{Conclusion}

In summary, our study was the first to construct the predicted miRNA-mRNA/IncRNA interaction networks that were associated with gonadal development in polyploid fishes. We identified many DE mRNAs, DE IncRNAs, and DE miRNAs through screening of different developmental stages and constructed ceRNA effector networks based on the effector relationships of mRNA-miRNA and IncRNA-miRNA. At the same time, the upregulated and down-regulated DE mRNAs at different developmental stages were annotated and analysed. Most of these RNAs were associated with gonadal development or meiosis. Many IncRNAs that are associated with gonadal development in fishes were discovered and identified. Their specific functions require future verification. The regulatory mechanism of triploidization on sterility was illuminated at the transcriptional or posttranscriptional level. In this study, we examined the effector relationships of the coding and non-coding RNAs during gonadal development in fishes at the whole genome level, and paved the way for further reductionist investigations and applications on acquaculture.

\section{Methods}

\section{Ethics statement}

All experiments were performed according to the European Communities Council Directive (86/609/EEC) norms and approved by the Animal Husbandry Department of Heilongjiang Animal Care and Use Committee. All fishes involved in this research were bred following the guidelines of the Animal Husbandry Department of Heilongjiang, P.R. China. All efforts were made to minimise the pain.

\section{Gonadal sampling of rainbow trout}

Triploid all-female (XXX) and diploid all-female (XX) rainbow trouts were obtained from Bohai experimental station of Heilongjiang River Fisheries Research Institute (Harbin, China). The triploid all-female eggs were obtained from the normal female eggs (XX) fertilised with pseudo-male semen $(X X)$, and then the fertilised eggs were shocked by hydrostatic pressure. The ploidy of eggs was verified by Ploidy Analyser (Sysmex, Japan), the result of ploidy identification of gonad samples were as shown in Supplementary Fig. S1, and the triploid rate could reach up to $99 \%$. The embryos

Page $7 / 20$ 
were incubated at $12{ }^{\circ} \mathrm{C}$ from fertilisation until 24 days post-fertilisation (dpf) and transferred to a hatch pool at $12+0.2^{\circ} \mathrm{C}$. The fries were transferred to a cement pool when they were grown to achieve around $40 \mathrm{~g}$ weight. To minimize suffering of the fish, before tissue collection, fish were euthanized with an overdose of anaesthesia in MS-222 (Sigma, St. Louis, MO, USA) as reported previously (Chang et al., 2017). The gonadal part of the fry at $65 \mathrm{dpf}$ was isolated under an anatomic microscope. Gonadal samples from 3 diploid female (XX) fishes and 3 triploid female (XXX) fishes were obtained, and the gonadal tissues of $X X$ and $X X X$ at $180 \mathrm{dpf}$ and $600 \mathrm{dpf}$ were sampled, respectively. The sample size of each ploidy at each time point was 3 . All gonads were sampled and stored at $-80^{\circ} \mathrm{C}$ for further experiments.

\section{Sequencing and differential expression analysis of mRNAs and IncRNAs}

The Trizol reagent (Invitrogen, CA, USA) was used to extract the total RNA, and only RIN of number >7.0, the RNA samples would be kept for the next experiments. The Epicentre Ribo-Zero Gold Kit (Illumina, San Diego, USA) was used to deplete ribosomal RNA of the total RNA. Under high temperature, RNA fractions were fragmented into small pieces. The mRNA-Seq sample preparation kit (Illumina, San Diego, USA) was used for RNA fragments to reverse-transcription to construct the final cDNA library. The average size for the paired-end libraries was 300 bp ( \pm ), and then the paired-end sequencing was performed on an Illumina Hiseq 4000 following the vendor's recommended protocol. Firstly, transcripts that overlapped with the known mRNAs and transcripts shorter than 200 bp were discarded. Cutadapt was used to fastq quality control as following steps: (1) reads with Adaptor were removed; (2) reads containing N (N indicates that the base information cannot be determined) at a rate greater than $5 \%$ were removed; (3) low quality (the number of bases with quality value Q $\leq 10$ accounts for more than $20 \%$ of the whole read) reads were removed. The clean and filtered reads were aligned to the reference genome of Oncorhynchus mykiss (https://www.ncbi.nlm.nih.gov/genome/? term=Oncorhynchus\%20mykiss, Omyk_1.0) using HISAT (v2.0.4) (min_intron_length=20『max_intron_length=500000). The valid reads and the percentage of mapped reads for each sample were shown as in Supplementary Table. S1. Thereafter, we utilised CPC (Kong et al., 2007) and CNCI (Sun et al., 2013) to predict transcripts having coding potentials. All transcripts with a CPC score of $<-1$ and $\mathrm{CNCl}$ score of $<0$ were removed. The remaining transcripts were considered as IncRNAs. StringTie (Frazee et al., 2015) was used to estimate the expression level of mRNAs and IncRNAs by calculating FPKM (Punta et al., 2012), the parameter of StringTie was set as stringtie_abundance=1. The differentially expressed mRNAs and IncRNAs were selected with log2 (fold change) $>1$ or log2 (fold change) $<-1$ and FDR $<0.05$ as significant cutoff values using $R$ package Ballgown (Agarwal et al., 2015), the parameter was set as remove_low_copy=0. The RNA-Seq raw data were deposited into the repository of SRA-NCBI, the BioProject number was PRJNA606210, and the BioSample IDs were SAMN14091286- SAMN14091303.

\section{Sequencing and differential expression analysis of miRNAs}

The TruSeq Small RNA Sample Prep Kits (Illumina, San Diego, USA) was used to construct the cDNA libraries. The cDNA product was purified by gel electrophoresis. After recovery, the purified library was concentrated by ethanol precipitation. The cDNA library was validated by DNA-1000 chip (Agilent Technologies 2100 Bioanalyzer, Agilent, CA, USA). The sequencing of cDNA libraries was performed using Illumina Hiseq 2500 through single-read strategy $(1 \times 50 \mathrm{bp})$. Raw reads were subjected to the ACGT101-miR tool (LC Sciences, Houston, Texas, USA) to remove adapter dimers, junk, low complexity, common RNA families (rRNA, tRNA, snRNA, snoRNA) and repeats (Ambady et al., 2012). Two aligners (Bowtie and Megablast) were used by ACGT101-miR, and their corresponding parameters were set as the following: Bowtie was used for reads to align against the mRNA sequence of Oncorhynchus mykiss

(ftp://ftp.ncbi.nlm.nih.gov/genomes/all/GCF/002/163/495/GCF_002163495.1_Omyk_1.0/GCF_002163495.1_Omyk_1.0_cds_from_genomic.fna.gz), Rfam, Repbase and miRBase 22.0 database setting seed length = 16 and mismatch $=1$. Megablast was used to align pre-miRNAs against the reference genome applying a $90 \%$ identity threshold. The unique sequences mapping to miRNAs' annotated loci on the genome of 0 . mykiss were identified as known miRNAs. The unique sequences mapping to the other arm of 0 . mykiss known pre-miRNAs, opposite to the annotated mature miRNA-containing arm, were considered to be novel 5p- or 3p-derived miRNA candidates. The remaining sequences were mapped to other selected species precursors (with the exclusion of the genome of 0 . mykiss) in miRBase 22.0, and the mapped pre-miRNAs were further aligned against the genome of 0 . mykiss to determine their genomic locations. The above two we defined as known miRNAs. The unmapped sequences were aligned against the genome of 0 . mykiss, and the hairpin RNA structure-containing sequences were predicted from the flank 80 nt sequences using RNAfold software (http://rna.tbi.univie.ac. at/cgi-bin/RNAfold.cgi). The criteria for secondary structure prediction were: (1) number of nucleotides in one bulge in stem ( $\leq 12)(2)$ number of base pairs in the stem region of the predicted hairpin ( $\geq 16)(3)$ cutoff of free energy (kCal/mol $\leq-15)(4)$ length of hairpin (up and down stems + terminal loop $\geq 50$ ) (5) length of hairpin loop ( $\leq 20)$. (6) number of nucleotides in one bulge in mature region ( $\leq 8)$ (7) number of biased errors in one bulge in mature region $(\leq 4)(8)$ number of biased bulges in mature region ( $\leq 2)(9)$ number of errors in mature region $(\leq 7)(10)$ number of base pairs in the mature region of the predicted hairpin $(\geq 12)(11)$ percent of mature in stem ( $\geq 80)$. Finally, the effective sequences of miRNAs were further analyzed. The differential expression of the miRNAs based on normalised read counts were assessed through Student's t-test, setting a significance threshold of 0.05 in each performed test.

\section{Construction of ceRNA network of miRNA-mRNA/IncRNA}

The DE IncRNAs and 3' UTR sequences of DE mRNAs were predicted as candidate miRNA targets using TargetScan (Agarwal et al., 2015) and miRanda (Betel et al., 2010). The threshold value of TargetScan for seed length scanned against the IncRNAs and 3' UTRs of mRNAs database was

Page $8 / 20$ 
context score percentile $>50$, and the threshold free energy $(\mathrm{kcal} / \mathrm{mol})$ of miRanda was established as Max_Energy<-10. The miRNA-target selection was performed according to the intersection between the two tools. These IncRNAs are regarded as competing endogenous RNAs (ceRNA) to bind miRNA competitively and affect mRNA (miRNA target gene) expression indirectly. The output of the ad-hoc perl scripts of DE miRNA-mRNA and miRNA-IncRNA of different developmental period were as shown in Supplementary Table. S2 and Table. S3. The ceRNA regulatory cascades (miRNAmRNA / IncRNA) were built by local perl scripts. The information including all of the above interaction was imported into Cytoscape (3.7.1) to construct a predicted miRNA-mRNA/IncRNA interaction network.

\section{Functional analysis of DE mRNA}

Gene Ontology (GO) enrichment analysis and Kyoto Encyclopaedia of Genes and Genomes (KEGG) pathway analyses were performed on the predicted target DE mRNAs of DE miRNA and DE IncRNAs in the ceRNA network, and DE mRNAs of different developmental period. For the nonmodel species, the specific steps of functional analysis were as follows: Firstly, the transcripts of the reference Oncorhynchus mykiss genome were used to align against the GO (go_20160702) and KEGG (release_78.1) database by Diamond with the parameter of E-value=1*E-5 (Buchfink et al., 2015). The comparison results of all transcripts for each gene which were integrated and removed duplicate were as the functional annotations of GO and KEGG. The local scripts were used for enrichment analysis. Then, the number of significantly DE genes (S gene number), the number of background genes (B gene number), the number of total significantly DE genes (TS gene number) and the number of total background genes (TB gene number) for each GO term/KEGG pathway were counted by GO and KEGG functional annotations. Pval was obtained by hypergeometric test. Both GO terms and KEGG pathways were considered to be significantly enriched with $\mathrm{FDR}<0.05$.

\section{Validation of RNAs in the network by real-time PCR}

Real-time PCR was performed for validation of the RNAs involved in the RNA-Seq. The same RNA samples were used for deep sequencing. A total of 10 IncRNAs, 12 mRNAs, and 11 miRNAs were detected from the real-time PCR expression. The qRT-PCR primers are shown in the Supplementary Table S4. For IncRNAs and mRNAs, the PrimeScript RT Reagent Kit (Takara, Dalian, China) was used for reverse transcription. Real-time PCR was performed using FastStart Universal SYBR® Green Master Mix (Roche, Switzerland) using CFX96 C1000 touch Thermal Cycler (BIO-RAD, America). Beta-actin was used as the reference gene. As for miRNAs, All-in-One ${ }^{\text {TM }}$ miRNA qRT-PCR Detection Kit (GeneCopoeia, America) was used for RNA reverse transcription and real-time PCR. The miRNA expression was normalised by the expression of 18s rRNA. All experiments were strictly performed following the instructions. The $\mathrm{Ct}$ values were measured, and the value of target sequence normalised to reference sequence was calculated as $2^{-\triangle \Delta C t}$. One-way ANOVA was used to assess the differences between the different groups. Statistical analysis was performed using SPSS 13.0 for Microsoft Windows. The significance was expressed as a $P$-value of $<0.05$.

\section{Abbreviations}

DEGs: Differentially expressed genes; cDNA: Complementary; RNA: Ribonucleic acid; mRNA: Messenger RNA; miRNA: microRNAs; IncRNA: Iong noncoding RNA; RNA-Seq: RNA-sequencing; FDR: False discovery rate; dpf: days post fertilisation.

\section{Declarations}

\section{Ethics approval and consent to participate}

All experiments were performed according to the European Communities Council Directive (86/609/EEC) norms and approved by the Animal Husbandry Department of Heilongjiang Animal Care and Use Committee.

\section{Consent for publication}

Not applicable.

\section{Availability of data and materials}

Specific data and local scripts used herein and the gtf and fasta files from the newly identified MSTRG IncRNAs and miRNAs can be provided upon readers request. The cytoscape files for networks could be provided upon request.

\section{Competing interests}

The authors declare that they have no competing interests. 


\section{Funding}

This study was supported by grants from the National Key R\&D Program of China (2018YFD0900200), the China Agriculture Research System (CARS-46) and Central Public-interest Scientific Institution Basal Research Fund囚CAFS(NO. 2020TD32). The funding agency played no part in study design, data collection and analysis, decision to publish, or preparation of the manuscript.

\section{Author's contributions}

1. H. designed and performed the experiments; G. X. analysed the data and checked the manuscript; W. G. and Z. Y. cultured and sampled the fish; E. L. drafted the article; T. H. and B. W. reviewed the manuscript. All authors contributed to the manuscript at various stages. All authors have read and approved the manuscript.

\section{Acknowledgements}

We thank all individuals who participated in this study.

\section{References}

1. Abramov, R., Fu, G., Zhang, Y., \& Peng, C. Expression and regulation of miR-17a and miR-430b in zebrafish ovarian follicles. Gen Comp Endocrinol. 2013;188: 309-315. https://doi.org/10.1016/j.ygcen.2013.02.012.

2. Agarwal, V., Bell, G. W., Nam, J. W., \& Bartel, D. P. Predicting effective microRNA target sites in mammalian mRNAs. Elife. 2015;4:e05005. https://doi.org/10.7554/ eLife.05005.001.

3. Ambady, S., Wu, Z., \& Dominko, T. Identification of novel microRNAs in Xenopus laevis metaphase II arrested eggs. Genesis. 2012;50(3):286-299. https://doi.org/ 10.1002/dvg.22010.

4. Barak Y, Liao D, He W, et al. Effects of peroxisome proliferator-activated receptor delta on placentation, adiposity, and colorectal cancer. Proceedings of the National Academy of Sciences. 2002;99(1):303-308. https://doi.org/10.1073/pnas. 012610299.

5. Benfey, T., J. The physiology and behavior of triploid fishes. Reviews in Fisheries Science. 1999;7:45-48. https://doi.org/10.1080/10641269991319162.

6. Betel D, Koppal A, Agius P, et al. Comprehensive modeling of microRNA targets predicts functional non-conserved and non-canonical sites. Genome Biology, 2010;11(8): R90. https://doi.org/10.1186/gb-2010-11-8-r90.

7. Bogacka I, Kurzynska A, Bogacki M, \& Chojnowska K. Peroxisome proliferator-activated receptors in the regulation of female reproductive functions. Folia Histochemica Cytobiologica. 2015;53(3): 189-200. https://doi.org/10.5603/fhc.a 2015.0023.

8. Braasch, I., et al. Corrigendum: the spotted gar genome illuminates vertebrate evolution and facilitates human teleost comparisons. Nature Genetics. 2016;48(6): 427-437. https://doi.org/10.1038/ng0616-700c.

9. Brown, J. B., Boley, N., Eisman, R., et al. Diversity and dynamics of the Drosophila transcriptome. Nature. 2014;512(7515):393-399. https://doi.org/10.1038/nature12962.

10. Buchfink, B., Xie, C., \& Huson, H. D. Fast and sensitive protein alignment using DIAMOND. Nature Methods. 2014;12(1):59-60. https://doi.org/10.1038/nmeth.3176.

11. Chang J, Niu H X, Jia Y D, et al. Effects of dietary lipid levels on growth, feed utilization, digestive tract enzyme activity and lipid deposition of juvenile Manchurian trout, Brachymystax lenok (Pallas)[J]. Aquaculture Nutrition, 2017, 24(2). https://10.1111/anu.12598.

12. Chassot, A. A., Gregoire, E. P., Lavery, R., Taketo, M. M., de Rooij, D. G., Adams, I. R., \& Chaboissier, M. C. RSPO1/beta-catenin signaling pathway regulates oogonia differentiation and entry into meiosis in the mouse fetal ovary. PLoS One. 2011;6(10): e25641.

https://doi.org/10.1371/journal.pone.0025641.

13. Chassot A A, Le Rolle M, Jourden M, Taketo M M, Ghyselinck N B, \& Chaboissier M C. Constitutive WNT/CTNNB1 activation triggers spermatogonial stem cell proliferation and germ cell depletion. Developmental Biology. 2017;426(1):17-27.

https://doi.org/10.1016/j.ydbio.2017.04.010.

14. Chassot, A. A., Ranc, F., Gregoire, E. P., Roepers-Gajadien, H. L., Taketo, M. M., Camerino, G., de Rooij, D. G., Schedl, A. \& Chaboissier, M. C. Activation of beta-catenin signaling by Rspo1 controls differentiation of the mammalian ovary. Hum Mol Genet. 2008;17(9):1264-1277. https://doi.org/10.1093/hmg/ddn016.

15. Cheng, Y., Feng, Y., Jansson, L., Sato, Y., Deguchi, M., Kawamura, K., \& Hsueh, A. J. Actin polymerization-enhancing drugs promote ovarian follicle growth mediated by the Hippo signaling effector YAP. FASEB J. 2015;29(6):2423-2430. https://doi.org/ 10.1096/fj.14-267856.

16. Chen Min, Wang Hong, Li Xiangdong,et al. PLIN1 deficiency affects testicular gene expression at the meiotic stage in the first wave of spermatogenesis. Gene. 2014;543(2):212-219. https://doi.org/10.1016/j.gene.2014.04.021.

Page $10 / 20$ 
17. Chourrout, D., et al. Production of second generation triploid and tetraploid rainbow trout by mating tetraploid males and diploid femalesPotential of tetraploid fish. Theoretical and Applied Genetics. 1986;72(2):193-206. https://doi.org/10.1007/ bf00266992.

18. Comai L. The advantages and disadvantages of being polyploid. Nat Rev Genet. 2005;6(11):836-846. https://doi.org/10.1038/nrg1711.

19. Cifuentes, M., Grandont, L., Moore, G., Chevre, A. M. \& Jenczewski, E. Genetic regulation of meiosis in polyploid species: new insights into an old question. New Phytologist. 2010;186(1):29-36. https://doi.org/10.2307/27797519.

20. Diter, A., et al. Gene segregation in induced tetraploid rainbow trout: genetic evidence of preferential pairing of homologous chromosomes. Genome. 1988;30(4):547-553. https://doi.org/10.1016/0141-0229(88)90030-0.

21. Feist, G. \& Schreck, C. B. Brain-pituitary-gonadal axis during early development and sexual differentiation in the rainbow trout, Oncorhynchus mykiss. Gen Comp Endocrinol, 1996;102(3):394-409. https://doi.org/10.1006/gcen.1996.0083.

22. Florencia X. Santiago Valtierra, et.al.. Elov/4 and Fa2h expression during rat spermatogenesis: a link to the very-long-chain PUFAs typical of germ cell sphingolipids. Journal of Lipid Research. 2018;59(7):1175-1189. https://doi.org/ 10.1194/jlr.M081885.

23. Frazee, A. C., Pertea, G., Jaffe, A. E., Langmead, B., Salzberg, S. L. \& Leek, J. T. Ballgown bridges the gap between transcriptome assembly and expression analysis. Nat Biotechnol. 2015;33(3):243-246. https://doi.org/10.1038/nbt.3172.

24. Fujihara, M., Yamamizu, K., Wildt, D. E., \& Songsasen, N. Expression pattern of matrix metalloproteinases changes during folliculogenesis in the cat ovary. Reprod Domest Anim. 2016;51(5):717-725. https://doi.org/10.1111/rda.12736.

25. Gu, Y., Zhang, L., \& Chen, X. Differential expression analysis of Paralichthys olivaceus microRNAs in adult ovary and testis by deep sequencing. Gen Comp Endocrinol. 2014;204:181-184. https://doi.org/10.1016/j.ygcen.2014.05.019.

26. Guo, L., Zhao, Y., Yang, S., Zhang, H., \& Chen, F. An integrated analysis of miRNA, IncRNA, and mRNA expression profiles. Biomed Res Int. 2014;345605. https://doi.org/10.1155/2014/345605

27. Halder, G., \& Johnson, R. L. Hippo signaling: growth control and beyond. Development. 2011;138(1): 9-22. https://doi.org/10.1242/dev.045500.

28. Guo, X., et al. All-triploid Pacific oysters (Crassostrea gigas Thunberg) produced by mating tetraploids and diploids. Aquaculture. 1996;142:149161. https://doi.org/ 10.1016/0044-8486(95)01243-5.

29. Herrera, L., Ottolenghi, C., Garcia-Ortiz, J. E., Pellegrini, M., Manini, F., Ko, M. S., Nagaraja, R., Forabosco, A., \& Schlessinger, D. Mouse ovary developmental RNA and protein markers from gene expression profiling. Dev Biol. 2005;279(2): 271-290. https://doi.org/10.1016/j.ydbio.2004.11.029.

30. Huang $T$, Sun $H$, Wang $Y, X u$ G, Wang $X$, \& Han Y. Effect of follicle cell autophagy on gonadal development of triploid female rainbow trout (Oncorhynchus mykiss). Fish Physiol Biochem. 2017;44(1):185-196. https://doi.org/10.1007/s10695-017-0423-7.

31. Jaillon, 0., et al. Genome duplication in the teleost fish Tetraodon nigroviridis reveals the early vertebrate proto-karyotype. Nature. 2004;431:946957. https://doi.org/ 10.1038/nature03025.

32. Kang, L., Yang, C., Wu, H., Chen, Q., Huang, L., Li, X., Tang, H., \& Jiang, Y. miR-26a-5p regulates TNRC6A Expression and Facilitates Theca Cell Proliferation in Chicken Ovarian Follicles. DNA Cell Biol. 2017;36(11):922-929. https://doi.org/ 10.1089/dna.2017.3863.

33. Kawamura, K., Cheng, Y., Suzuki, N., Deguchi, M., Sato, Y., Takae, S., Ho, C. H., Kawamura, N., Tamura, M., Hashimoto, S., Sugishita, Y., Morimoto, Y., Hosoi, Y., Yoshioka, N., Ishizuka, B., \& Hsueh, A. J. Hippo signaling disruption and Akt stimulation of ovarian follicles for infertility treatment. Proc Natl Acad Sci USA. 2013;110(43):17474-17479. https://doi.org/10.1073/pnas.1312830110.

34. Kawashima, I., \& Kawamura, K. Regulation of follicle growth through hormonal factors and mechanical cues mediated by Hippo signaling pathway. Syst Biol Reprod Med. 2017;64(1):3-11. https://doi.org/10.1080/19396368.2017.1411990.

35. Kong, L., Zhang, Y., Ye, Z. Q., Liu, X. Q., Zhao, S. Q., Wei, L., \& Gao, G. CPC: assess the protein-coding potential of transcripts using sequence features and support vector machine. Nucleic Acids Res. 2007;35(Web Server issue):W345-349. https://doi.org/10.1093/nar/gkm391.

36. Laiho, A., Kotaja, N., Gyenesei, A., \& Sironen, A. Transcriptome profiling of the murine testis during the first wave of spermatogenesis. PLoS One. 2013;8(4): e61558. https://doi.org/10.1371/journal.pone.0061558.

37. Lau, E. (2014). Non-coding RNA: Zooming in on IncRNA functions. Nat Rev Genet. 2014;15(9):574-575. https://doi.org/10.1038/nrg3795.

38. Leitch, A.R. \& Leitch, I.J. Genomic plasticity and the diversity of polyploid plants. Science. 2008;320(5875):481-483. https://doi.org/10.1126/science.1153585.

39. Lian, C., Zuo, Q., Li, D., Zhang, L., Ahmed, M. F., Xiao, T., Tang, B., Wang, Y., Jin, K., Zhang, Y. \& Li, B. Basing RNA-seq explored the regulatory mechanism of the carbohydrate metabolism pathways during chicken male germ cell differentiation. In Vitro Cell Dev Biol Anim. 2015;51(7):690-696. https://doi.org/10.1007/s11626-015-9874-2.

40. Mark, M., Jacobs, H., Oulad-Abdelghani, M., Dennefeld, C., Feret, B., Vernet, N., Codreanu, C. A., Chambon, P., \& Ghyselinck, N. B. STRA8-deficient spermatocytes initiate, but fail to complete, meiosis and undergo premature chromosome condensation. J Cell Sci. 2008;121(19):3233-3242. https://doi.org/10.1242/jcs.035071

41. Meyer A. \& van De. Peer. Y. From 2R to 3R: evidence for a fish-specific genome duplication (FSGD). BioEssays. 2005;27:937945.https://doi.org/10.1002/bies.20293. 
42. Navarro-Martin, L., Galay-Burgos, M., Piferrer, F., \& Sweeney G. Characterisation and expression during sex differentiation of Sox 19 from the sea bass Dicentrarchus labrax. Comp Biochem Physiol B Biochem Mol Biol. 2012;163(3-4):316-323. https://doi.org/10.1016/j.cbpb.2012.08.004.

43. Pan, D. Hippo signaling in organ size control. Genes Dev. 2007;21(8):886-897. https://doi.org/10.1101/gad.1536007.

44. Pati, D., \& Habibi, H. R. Direct action of GnRH variants on goldfish oocyte meiosis and follicular steroidogenesis. Mol Cell Endocrinol. 2000;160(1-2):75-88. https://doi. org/10.1016/S0303-7207(99)00250-6.

45. Punta, M., Coggill, P. C., Eberhardt, R. Y., Mistry, J., Tate, J., Boursnell, C., Pang, N., Forslund, K., Ceric, G., Clements, J., Heger, A., Holm, L., Sonnhammer, E. L., Eddy, S. R., Bateman, A., \& Finn, R. D. The Pfam protein families database. Nucleic Acids Res. 2012;40(Database issue):D290-301. https://doi.org/10.1093/ nar/gkr1065.

46. Song, Y. N., Shi, L. L., Liu, Z. Q., \& Qiu, G. F. Global analysis of the ovarian microRNA transcriptome: implication for miR-2 and miR-133 regulation of oocyte meiosis in the Chinese mitten crab, Eriocheir sinensis (Crustacea:Decapoda). BMC Genomics. 2014;15(1): 547. https://doi.org/10.1186/1471-2164-15-547.

47. Stenberg, P., \& Saura, A. Meiosis and its deviations in polyploid animals. Cytogenet Genome Res. 2013;140(2-4):185-203. https://doi.org/10.1159/000351731.

48. Sun, L., Luo, H., Bu, D., Zhao, G., Yu, K., Zhang, C., Liu, Y., Chen, R., \& Zhao, Y. Utilizing sequence intrinsic composition to classify protein-coding and long non-coding transcripts. Nucleic Acids Res. 2013;41(17):e166. https://doi.org/ 10.1093/nar/gkt646.

49. Taylor, D. H., Chu, E. T., Spektor, R., \& Soloway, P. D. Long non-coding RNA regulation of reproduction and development. Mol Reprod Dev. 2015;82(12):932-956. https://doi.org/10.1002/mrd.22581.

50. Tiwary, B.K., Kirubagaran, R., Raym, A.K. The biology of triploid fish. Rev Fish Biol Fish. 2004;14:391-402. https://doi.org/10.1007/s11160-0048361-8.

51. Van de Peer Y, et al. The evolutionary significance of ancient genome duplications. Nat Rev Genet. 2009;10:725-32. https://doi.org/10.1038/nrg2600.

52. Van de Peer Y, et al. The evolutionary significance of polyploidy. Nat Rev Genet. 2017;18:411-424. https://doi.org/10.1038/nrg.2017.26.

53. Wang, F., Jia, Y., Wang, P., Yang, Q., Du, Q., \& Chang, Z. Identification and profiling of Cyprinus carpio microRNAs during ovary differentiation by deep sequencing. BMC Genomics. 2017;18(1): 333. https://doi.org/10.1186/s12864-017-3701-y.

54. Wen, K., Yang, L., Xiong, T., Di, C., Ma, D., Wu, M., Xue, Z., Zhang, X., Long, L., Zhang, W., Zhang, J., Bi, X., Dai, J., Zhang, Q., Lu, Z. J., \& Gao, G. Critical roles of long noncoding RNAs in Drosophila spermatogenesis. Genome Res. 2016;26(9): 1233-1244. https://doi.org/10.1101/gr.199547.115.

55. Wu, Z., Liu, X., Liu, L., Deng, H., Zhang, J., Xu, Q., Cen, B., \& Ji, A. Regulation of IncRNA expression. Cell Mol Biol Lett. 2014;19(4):561-575. https://doi.org/ 10.2478/s11658-014-0212-6.

56. Xiao, J., Zhong, H., Zhou, Y., Yu, F., Gao, Y., Luo, Y., Tang, Z., Guo, Z., Guo, E., Gan, X., Zhang, M., \& Zhang, Y. Identification and characterization of microRNAs in ovary and testis of Nile tilapia (Oreochromis niloticus) by using solexa sequencing technology. PLoS One. 2014;9(1):e86821. https://doi.org/10.1371/journal.pone. 0086821.

57. Xu, G., Huang, T., Jin, X., Cui, C., Li, D., Sun, C., Han, Y., \& Mu, Z. Morphology, sex steroid level and gene expression analysis in gonadal sex reversal of triploid female (XXX) rainbow trout (Oncorhynchus mykiss). Fish Physiol Biochem. 2016;42(1):193-202. https://doi.org/10.1007/s10695-015-0129-7.

58. Yang, F., Guan, J., Li, R., Li, X., Niu, J., Shang, R., Qi, J., \& Wang, X. miR-1388 regulates the expression of nectin2l in Paralichthys olivaceus. Comp Biochem Physiol Part D Genomics Proteomics. 2018;28:9-16. https://doi.org/10.1016/j.cbd. 2018.05.003.

59. Ye, W., Lv, Q., Wong, C. K., Hu, S., Fu, C., Hua, Z., Cai, G., Li, G., Yang, B. B., \& Zhang, Y. The effect of central loops in miRNA:MRE duplexes on the efficiency of miRNA-mediated gene regulation. PLoS One. 2008;3(3):e1719. https://doi.org/ 10.1371/journal.pone.0001719.

60. Yin, C., Zhang, J., Shi, Z., Sun, W., Zhang, H., \& Fu, Y. Identification and expression of the target gene emx2 of miR-26a and miR-26b in Paralichthys olivaceus. Gene. 2015;570(2): 205-212. https://doi.org/10.1016/j.gene.2015.06.030.

61. Zhang, J., Yu, P., Zhou, Q., Li, X., Ding, S., Su, S., Zhang, X., Yang, X., Zhou, W., Wan, Q., \& Gui, J. F. Screening and characterisation of sex differentiation-related long non-coding RNAs in Chinese soft-shell turtle (Pelodiscus sinensis). Sci Rep. 2018;8(1):8630. https://doi.org/10.1038/s41598-018-26841-3.

62. Zhang, X., Shi, J., Sun, Y., Zhu, Y., Zhang, Z., \& Wang, Y. (2019). Transcriptome analysis provides insights into differentially expressed genes and long noncoding RNAs involved in sex-related differences in Amur sturgeon (Acipenser schrenckii). Mol Reprod Dev. 2019;86(2):132-144. https://doi.org/10.1002/mrd.23065.

63. Zhang, Y. C., Liao, J. Y., Li, Z. Y., Yu, Y., Zhang, J. P., Li, Q. F., Qu, L. H., Shu, W. S. \& Chen, Y. Q. Genome-wide screening and functional analysis identify a large number of long noncoding RNAs involved in the sexual reproduction of rice. Genome Biol. $2014 ; 15(12): 512$. https://doi.org/10.1186/s13059-014-0512-1.

\section{Figures}



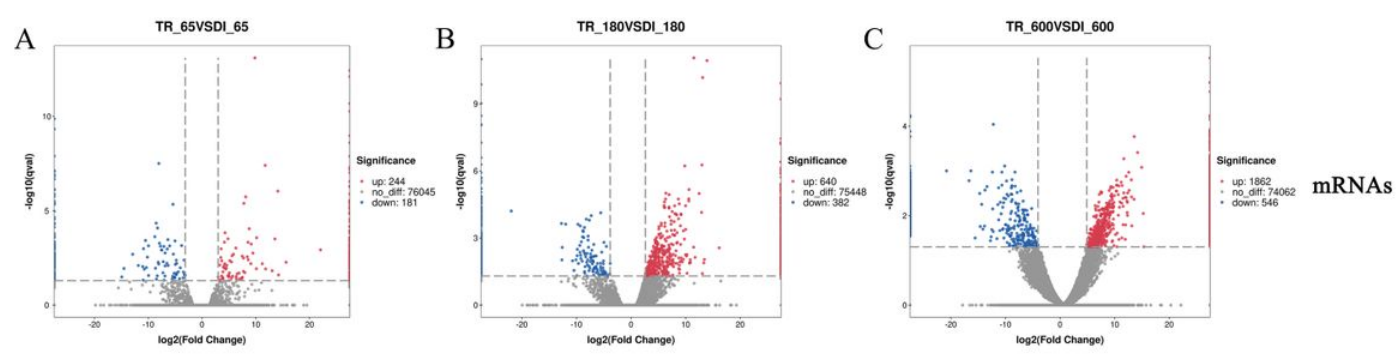

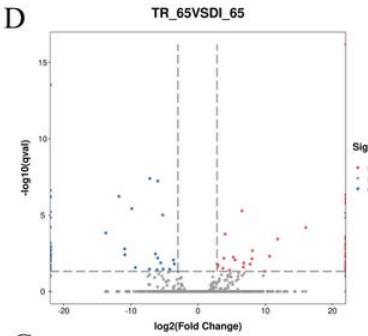

G

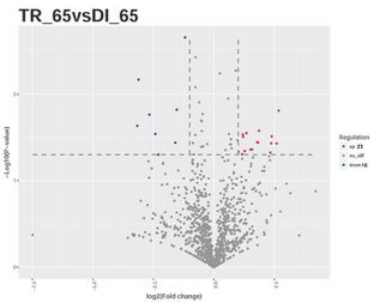

$\mathrm{J}$

Differentially Expressed mRNAs

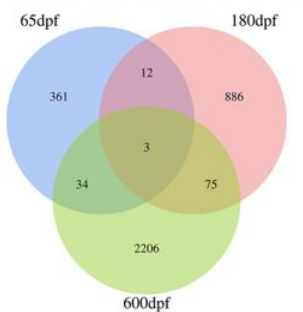

E

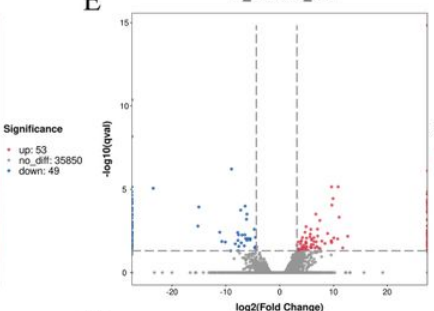

$\mathrm{H}$

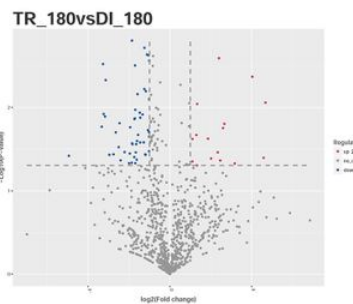

K

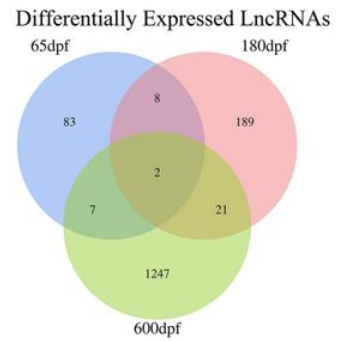

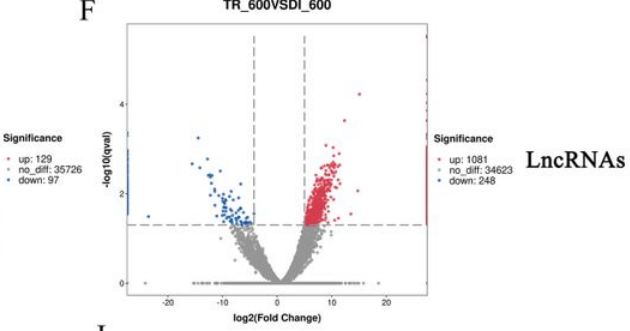

I

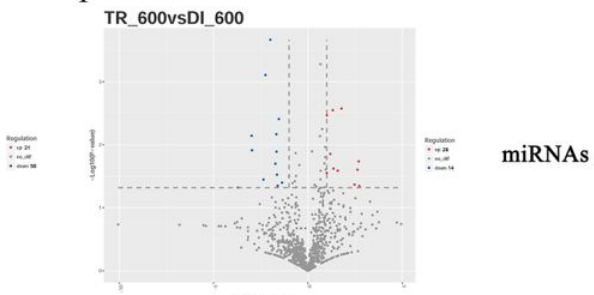

L Differentially Expressed miRNAs

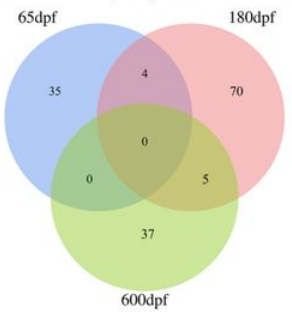

Figure 1

Identification of the overlapping DE mRNAs, DE IncRNAs, and DE miRNAs related to gonadal development. (A-C) Volcano plots of DE mRNAs in the triploid (XXX) and diploid (XX) gonads at $65 \mathrm{dpf}, 180 \mathrm{dpf}$, and $600 \mathrm{dpf}$. (D-F) Volcano plots for DE IncRNAs in the triploid (XXX) and diploid (XX) gonads at $65 \mathrm{dpf}, 180 \mathrm{dpf}$, and $600 \mathrm{dpf}$. (G-I) Volcano plots for DE miRNAs in the triploid (XXX) and diploid (XX) gonads at $65 \mathrm{dpf}, 180 \mathrm{dpf}$, and 600 dpf. (J) Venn diagram for the overlapping DE mRNAs of $65 \mathrm{dpf}, 180 \mathrm{dpf}$, and $600 \mathrm{dpf}$. (K) Venn diagram for the overlapping DE IncRNAs of $65 \mathrm{dpf}$, $180 \mathrm{dpf}$, and $600 \mathrm{dpf}$. (L) Venn diagram for the overlapping DE miRNAs of $65 \mathrm{dpf}, 180 \mathrm{dpf}$, and $600 \mathrm{dpf}$. 

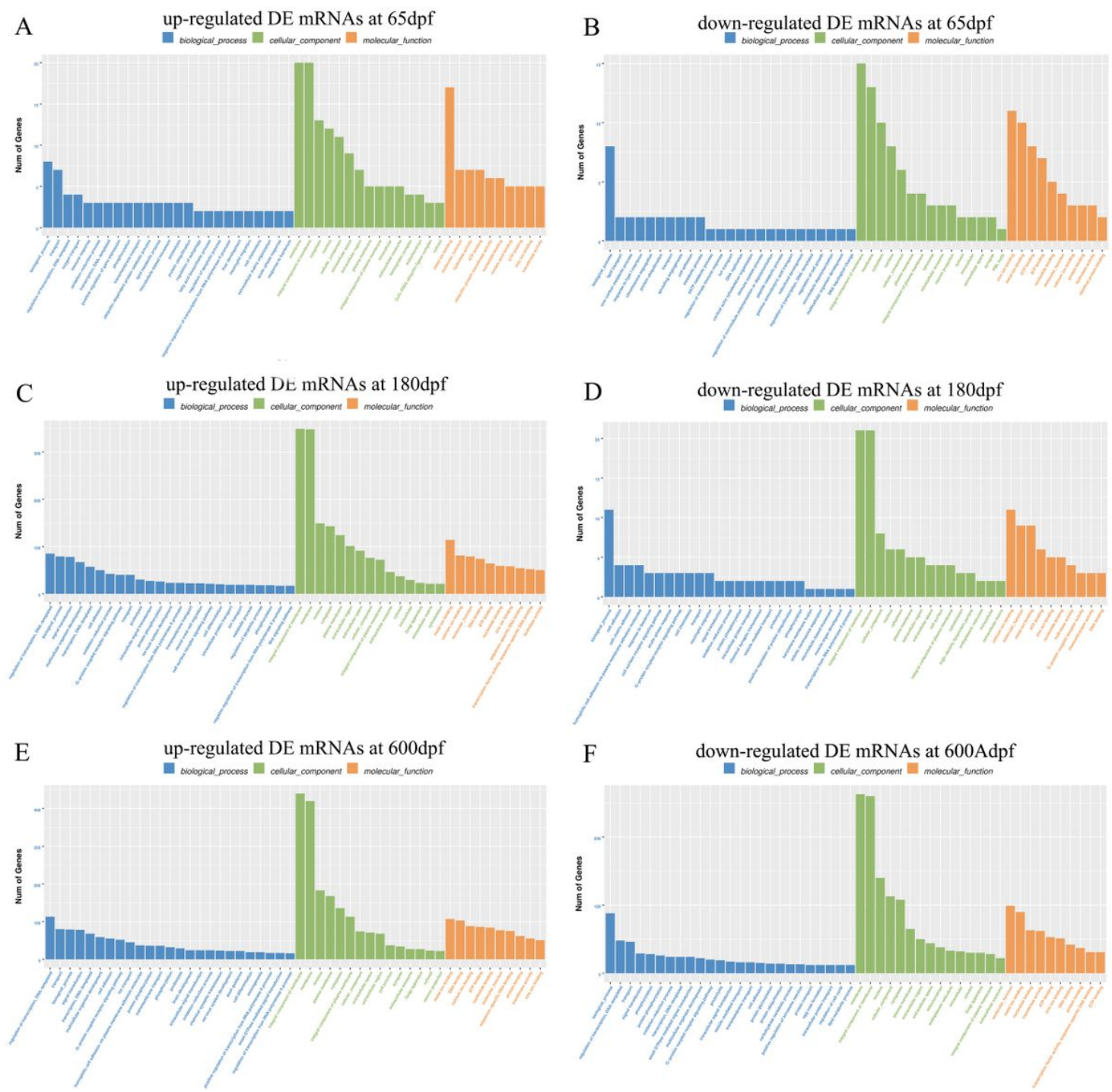

Figure 2

GO analysis of the differentially expressed mRNAs at each developmental point according to the up- and down- regulated DE mRNAs. (A) GO analysis of up-regulated DE mRNAs at $65 \mathrm{dpf}$. (B) GO analysis of down-regulated DE mRNAs at $65 \mathrm{dpf}$. (C) GO analysis of up-regulated DE mRNAs at 180 dpf. (D) GO analysis of down-regulated DE mRNAs at 180 dpf. (E) GO analysis of up-regulated DE mRNAs at 600 dpf. (F) GO analysis of downregulated DE mRNAs at $600 \mathrm{dpf}$. 

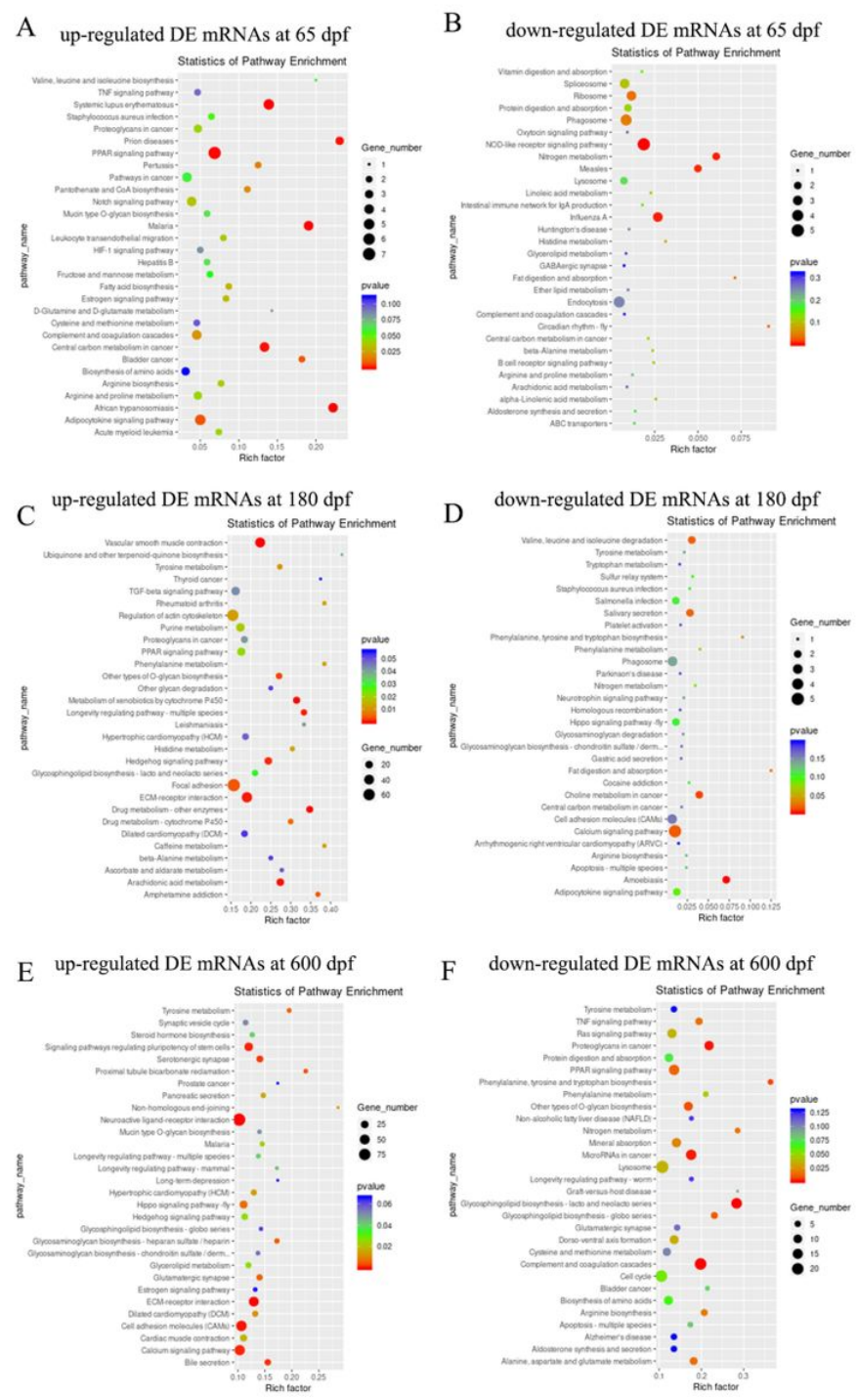

Figure 3

KEGG pathway analysis of the differentially expressed mRNAs at each developmental point according to the up- and down- regulated DE mRNAs. (A) KEGG analysis of up-regulated DE mRNAs at 65 dpf. (B) KEGG analysis of down-regulated DE mRNAs at 65 dpf. (C) KEGG analysis of up-regulated DE mRNAs at $180 \mathrm{dpf}$. (D) KEGG analysis of down-regulated DE mRNAs at $180 \mathrm{dpf}$. (E) KEGG analysis of up-regulated DE mRNAs at 600 dpf. (F) KEGG analysis of down-regulated DE mRNAs at $600 \mathrm{dpf}$. 


\section{GO Enrichment}

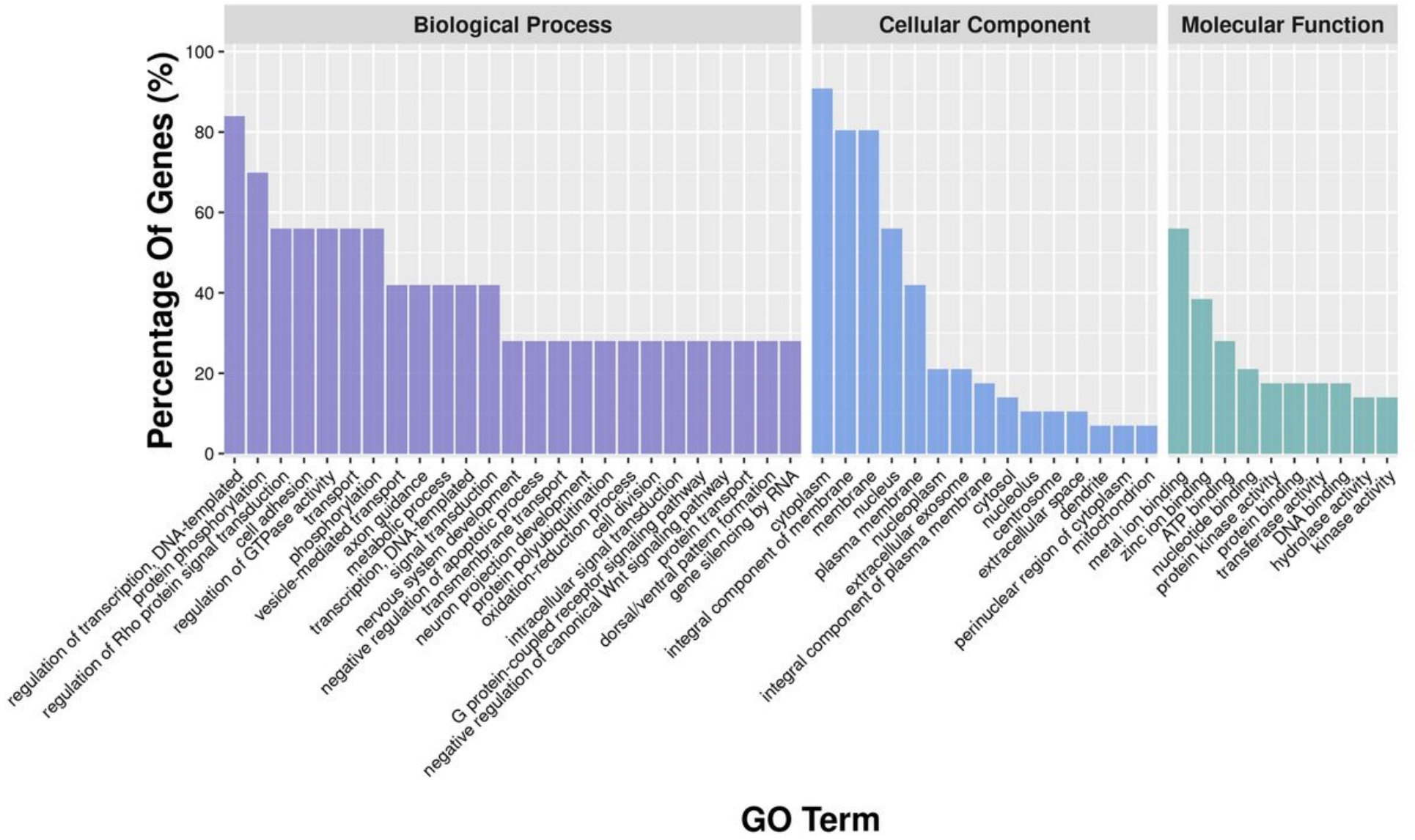

Figure 4

GO analysis of the overlapping DE mRNAs of $65 \mathrm{dpf}, 180 \mathrm{dpf}$, and $600 \mathrm{dpf}$. 75+12+3+34 DE mRNAs from Fig 1J. 
A

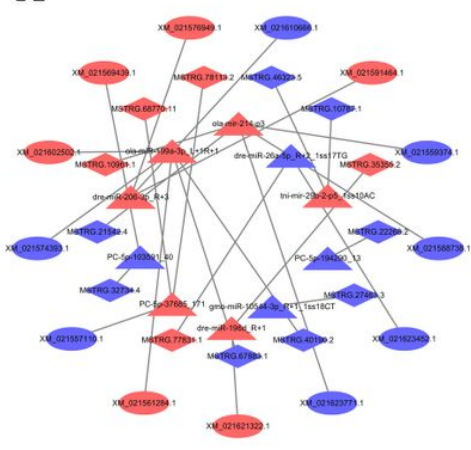

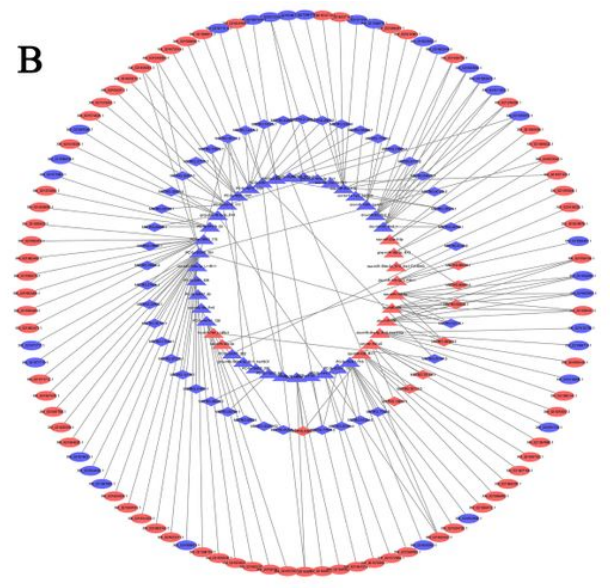

$\mathrm{C}$

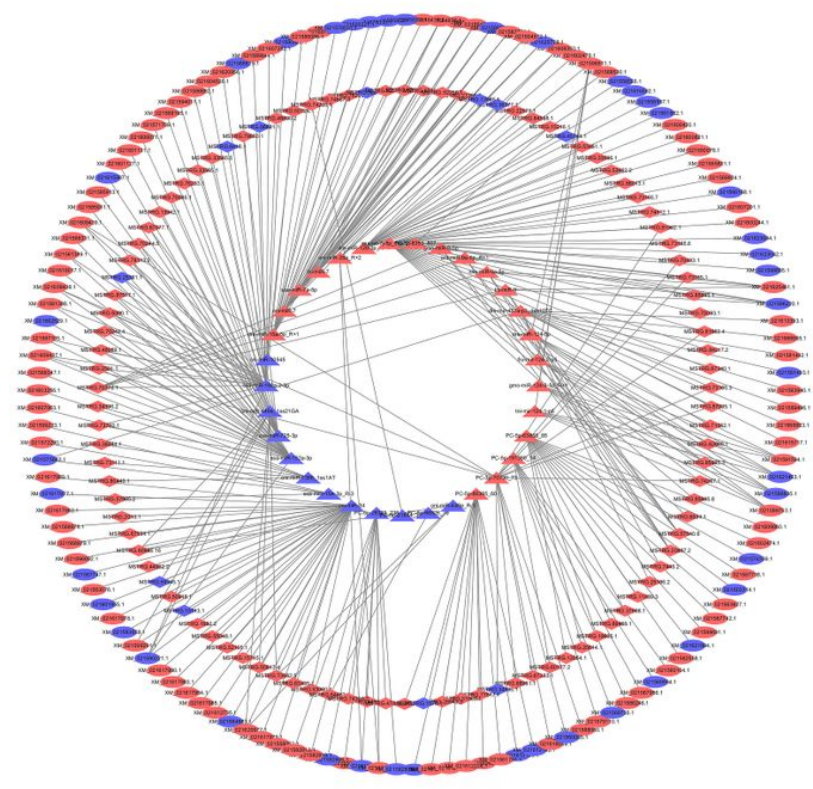

\section{Figure 5}

The predicted miRNA-mRNA/IncRNA interaction network of $65 \mathrm{dpf}(\mathrm{A}), 180 \mathrm{dpf}(\mathrm{B})$ and $600 \mathrm{dpf}(\mathrm{C})$. The ellipse represents mRNAs, the rectangle represents IncRNAs, and the triangle represents miRNAs. The colour of pink represents up-regulated nodes, and the colour of blue represents downregulated nodes. 


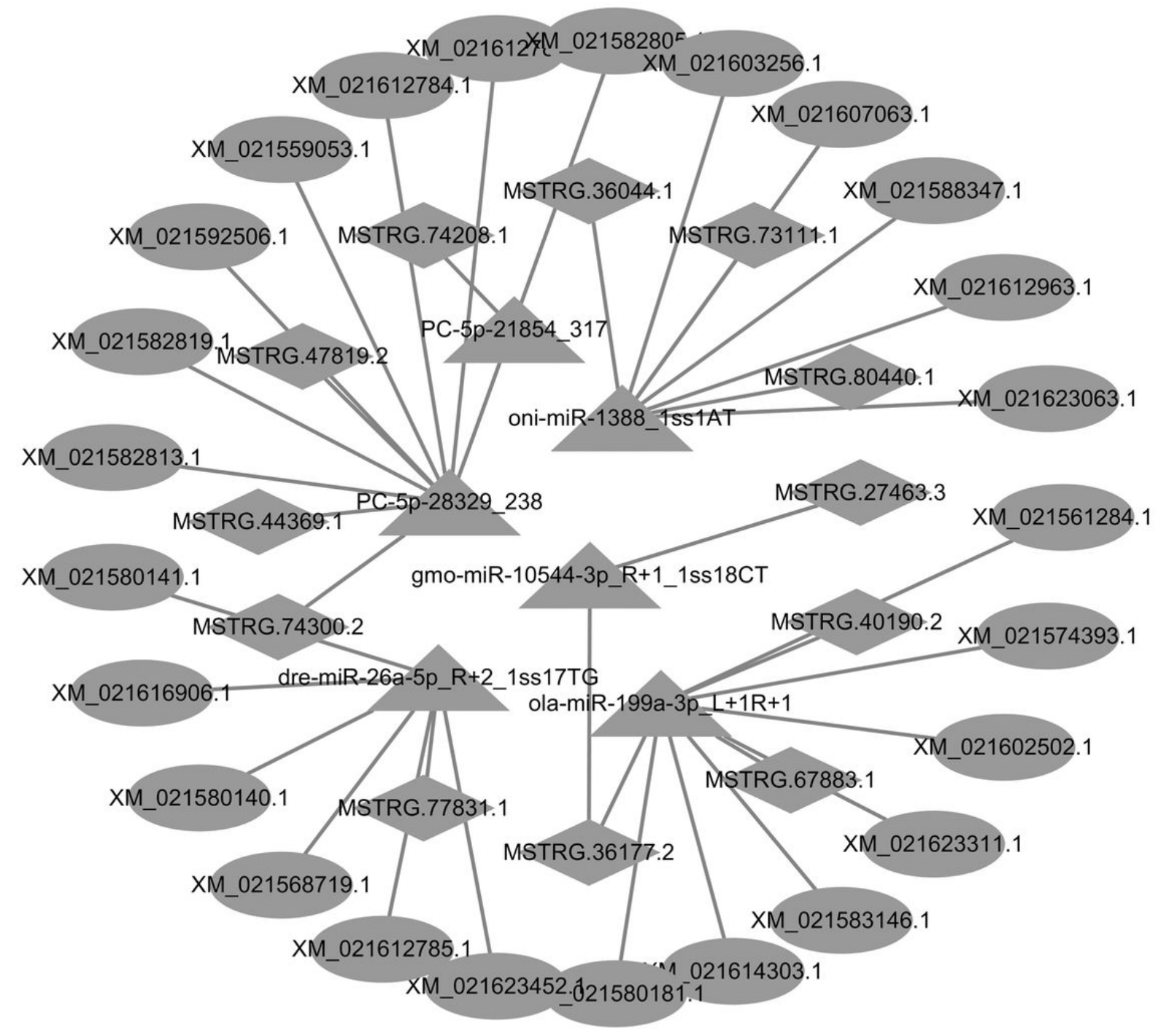

Figure 6

The predicted miRNA-mRNA/IncRNA interaction network based on overlapped DE RNAs from the three previously built networks for each timepoint in Figure 5. The network consisted of 6 DE miRNAs, 12 DE IncRNAs, and 25 DE mRNAs. The ellipse represents mRNAs, the rectangle represents IncRNAs, and the triangle represents miRNAs. 
A

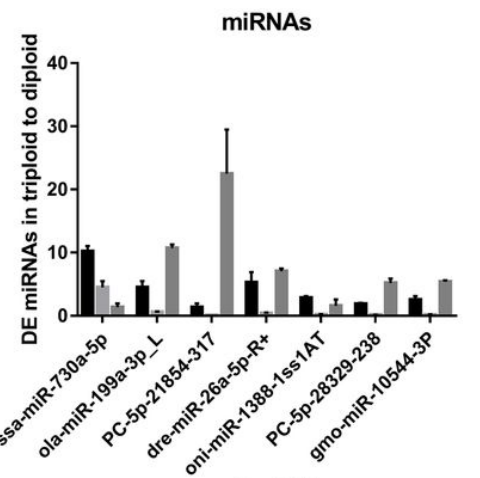

$65 \mathrm{dpf}$

$180 \mathrm{dpf}$

- $600 \mathrm{dpf}$

B

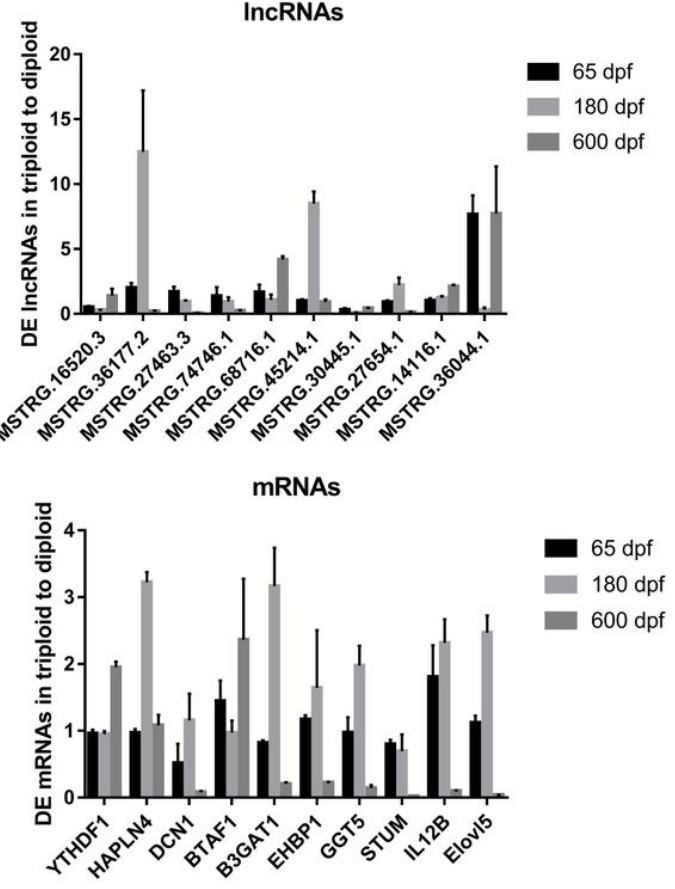

Figure 7

Validation of RNA expression by quantitative real-time PCR. (A) The expression of 7 miRNAs in the gonadal tissues of triploid (XXX) rainbow trout as compared to that of the diploid (XX) at $65 \mathrm{dpf}, 180 \mathrm{dpf}$, and $600 \mathrm{dpf}$. (B) The expression of 10 IncRNAs in the gonadal tissues of triploid rainbow trout as compared to that of the diploid at $65 \mathrm{dpf}, 180 \mathrm{dpf}$, and $600 \mathrm{dpf}$. (C) The expression of 10 mRNAs in the gonadal tissues of triploid (XXX) rainbow trout as compared to that of the diploid (XX) at $65 \mathrm{dpf}, 180 \mathrm{dpf}$, and $600 \mathrm{dpf}$. The values are represented as the means $\pm S E M$ for three individuals. The significance was expressed as a P-value of $<0.05$.

\section{Supplementary Files}

This is a list of supplementary files associated with this preprint. Click to download.

- Tables14.Docx

- Tables4.docx

- Tables1.docx

- Tables11.xlsx

- Tables13.xlsx

- TableS12.xIsx

- Figures1.jpg

- Figures2review.jpg

- TableS5.XLSX

- Tables7.XLSX 
- TableS2.XLSX

- TableS10.XLSX

- TableS3.XLSX

- TableS9.XLSX

- TableS8.XLSX

- TableS6.XLSX 